$16^{\text {th }}$ International Congress of Metrology, 09004 (2013)

DOI: $10.1051 /$ metrology/201309004

(c) Owned by the authors, published by EDP Sciences, 2013

\title{
Métrologie des matériaux pour la photonique et l'énergie
}

\author{
Jean-Remy Filtz ${ }^{1, a}$, Bruno Hay ${ }^{1}$, Frédérique Haloua ${ }^{1}$, Jacques Hameury and Jimmy Dubard ${ }^{1}$ \\ ${ }^{1}$ LCM - (LNE-Cnam), 29, Av. R. Hennequin, ZA Trappes - Elancourt, 78190 Trappes, France
}

\begin{abstract}
Materials Science is an extremely wide and complex field in physics and chemistry. Many academic institutes and public or private research laboratories implement research in that field. However, very few National Metrology Institutes have developed skills in that area. LNE one of these institutes has developed a research program for addressing the most important and critical needs. This paper is intended to highlight with examples the contribution of the laboratory for helping industry and society and therefore the interest to amplify the basic and applied research in thermophysical and optical properties metrology for materials.
\end{abstract}

\section{Introduction - Défis}

Depuis de nombreuses années le LNE est un membre actif dans le domaine de la métrologie des matériaux en chimie. Plus récemment, durant la dernière décennie, dans le domaine de la physique, il a entrepris un programme de recherche et développement avec pour objectif prioritaire de mieux répondre aux besoins industriels les plus importants et les plus critiques. En effet le LNE, promoteur européen des "Mesures et Références », est très sollicité par les changements forts opérés par la société et aussi pour accroître la compétitivité industrielle.

Cette présentation met ici l'accent sur les thématiques en lien avec les champs disciplinaires Thermique, Optique et Photonique, cœur de métier de l'un des pôles de développement du LNE.

La contribution du LNE au service de l'industrie et de la société est abordée au travers de plusieurs exemples concrets. L'objectif est de souligner tout l'intérêt d'amplifier avec vigueur la recherche fondamentale et appliquée en métrologie des propriétés thermo-physiques et optiques des matériaux

\section{Face aux enjeux industriels}

Pour répondre avec excellence et efficience aux défis nationaux et européens, trois familles d'actions de développement ont été entreprises au LNE. Elles visent à renforcer les moyens et compétences pour permettre à moyen et long terme d'atteindre un leadership en métrologie des propriétés thermiques et optiques des matériaux au niveau national et européen.

\footnotetext{
a Corresponding author: jean-remy.filtz@lne.fr
}

\subsection{Développement d'une plateforme}

Une nouvelle plateforme métrologique - MATIS (MATériaux pour l'Industrie et la Société) dédiée à la caractérisation des propriétés thermiques et optiques des matériaux a été construite (Figure 1).

Figure 1. Vue du laboratoire MAXWELL accueillant la Plateforme MATIS

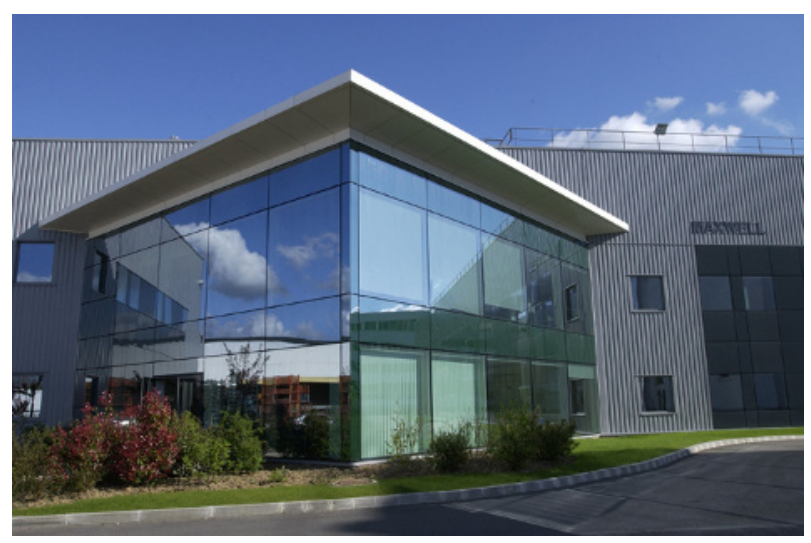

\subsection{Renforcement des compétences}

Les compétences du pôle Photonique et Energie du LNE ont été renforcées sur le versant des matériaux, plus particulièrement dans les domaines scientifiques et techniques suivants:

- Propriétés de transport thermique,

- Propriétés radiatives,

- Grandeurs calorifiques

- Propriétés optiques 


\subsection{Acteur d'un réseau européen}

Dans le cadre de la construction d'une infrastructure européenne en métrologie, le LNE pour la France, a proposé des initiatives de recherche en métrologie pour mieux satisfaire les besoins industriels et sociétaux.

Trois voies de développement sont menées en parallèle pour répondre aux enjeux :

- Renforcer la métrologie dédiée aux matériaux,

- Développer l'instrumentation la plus performante,

- Compléter le champ de connaissances.

Les développements scientifiques relatifs aux matériaux (solide, liquide, gaz) en cours dans les différents Instituts Nationaux de Métrologie, fourniront les solutions à moyen terme, par exemple pour des besoins ciblés dans le domaine de la production industrielle, pour développer de nouvelles technologies et aussi pour contribuer à la mise en pratique ou au développement des connaissances scientifiques plus fondamentales.

\section{Références nationales}

Les capacités de mesure de la plateforme MATIS offrent la palette de références nationales la plus complète en Europe pour réaliser des mesures et étalonnages sur une large plage de température $\left(\mathrm{de}-150{ }^{\circ} \mathrm{C}\right.$ jusqu'à $2500{ }^{\circ} \mathrm{C}$ en fonction des propriétés), pour les grandeurs suivantes :

\section{Propriétés thermiques et grandeurs calorimétriques :}

- diffusivité thermique,

- conductivité thermique

- dilatation thermique linéique,

- capacité thermique massique, ...

Propriétés radiatives :

- émissivité directionnelle spectrale,

- $\quad$ émissivité totale hémisphérique, ...

- facteurs de réflexion

\section{Propriétés optiques :}

- transmission spectrale régulière des filtres,

- transmission et réflexion régulières spectrales des matériaux (spectrophotométrie UV-visibleIR),

- indice de brillant,

- indice de réfraction de verres,

- références pour l'optique guidée (réflectance, linéarité, affaiblissement spectral, OLCR...)

Les moyens associés pour effectuer ces mesures constituent des références directement accessibles aux industriels pour assurer les missions de traçabilité au SI et de raccordement aux meilleurs niveaux d'incertitudes.

$\mathrm{Au}$ delà des moyens de base de cette structure, l'ambition du laboratoire est de compléter le dispositif en construisant de la valeur ajoutée technique et scientifique en phase avec les besoins et attentes industriels actuels. Les paragraphes qui suivent se proposent d'illustrer à partir de deux exemples choisis, des axes nouveaux de développement en cours.

\section{Métrologie et Sources d'énergie}

\subsection{Performances énergétiques du Gaz naturel}

Alors que la gestion des réseaux de transport et de distribution reste un service public non concurrentiel, le marché du gaz naturel en France a été libéralisé (marchés professionnels et domestiques) depuis 2007, afin de respecter les directives 98/30 du 22 juin 1998 et 2003/55 du 26 juin 2003, définissant les modalités relatives au marché intérieur du gaz naturel. En conséquence, le gaz naturel commercialisé en Europe pouvant provenir de différents pays, sa composition et donc son pouvoir calorifique supérieur peuvent varier d'une source d'approvisionnement à l'autre. Compte tenu des enjeux financiers, il est nécessaire de connaître avec une très faible incertitude les propriétés énergétiques de ces gaz naturels. Dans cette logique, le LNE, d'une part avec les soutiens de GdF-Suez et des pouvoirs publics et d'autre part dans la cadre d'un consortium de recherche européen GERG, a développé un calorimètre de référence pour déterminer le pouvoir calorifique supérieur (PCS) des composants du gaz naturel (méthane, éthane,...) avec une incertitude meilleure que $0,05 \%[1,2,3]$.

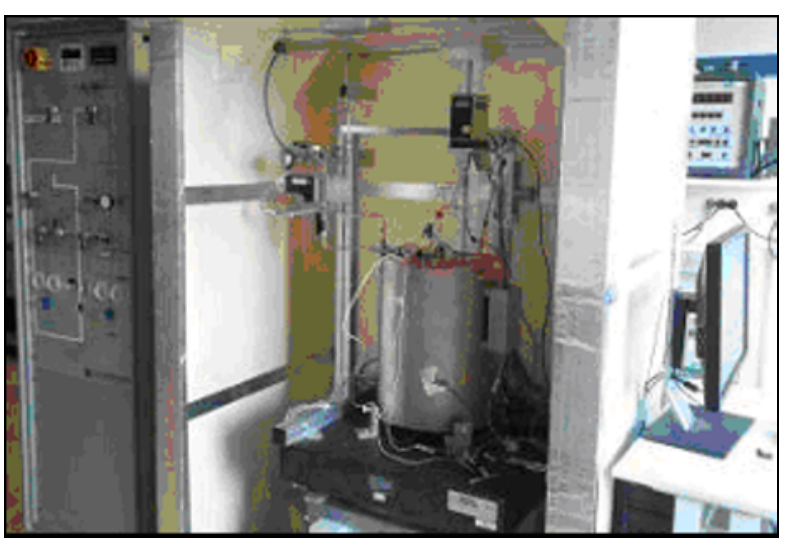

Figure 2. Gaz Naturel - Calorimètre de référence du LNE

Ces travaux permettent de contribuer à l'évolution de la normalisation menée au sein du WG 18 sur le référentiel IS0 6976.

\subsection{Performances énergétiques : Autres gaz}

Les travaux précédents ont permis d'acquérir des connaissances et de développer des moyens métrologiques parmi les plus performants en Europe pour la mesure du pouvoir calorifique supérieur de composants gazeux.

Par ailleurs, avec d'une part la volonté de diversifier, réduire la consommation des sources d'énergie primaire d'origine fossile (transition énergétique), et d'autre part de participer à un développement d'une société plus sure et durable (interchangeabilité des gaz dans les réseaux de distribution de gaz - "gas grids »), d'autres intérêts 
métrologiques liés aux performances énergétiques des gaz se sont révélés. L'expérience sur le gaz naturel a donc permis récemment de participer à un nouveau projet européen [4], visant en particulier à étendre les moyens d'expérimentation et les méthodologies de mesures à des gaz moins conventionnels, à savoir des gaz non-fossiles tels que des mélanges à base de méthane/dioxyde de carbone et sulfure d'hydrogène. Pour illustration on présente ci-dessous un exemple de résultat [5] obtenu avec un mélange ternaire.

Table 1. Exemple de PCS d'un mélange ternaire

\begin{tabular}{|c|c|c|c|c|}
\hline Composition & $\begin{array}{c}\text { PCS } \\
\text { (J.g-1) }\end{array}$ & $\begin{array}{c}\text { uPCS } \\
(\mathbf{J . g}-1)\end{array}$ & $\begin{array}{c}\text { uPCS } \\
\mathbf{( \% )}\end{array}$ & $\begin{array}{c}\text { Uc PCS } \\
(\mathbf{\%})(\mathbf{k}=\mathbf{2})\end{array}$ \\
\hline $\begin{array}{c}\text { Mélange } \\
\text { ternaire type } \\
\text { CH4/CO2/H2S }\end{array}$ & 14844.47 & 5.445 & 0.037 & 0.143 \\
\hline
\end{tabular}

L'objectif est ici de pouvoir travailler sur des mélanges avec des composants en proportion variable et d'étudier la répétabilité et la reproductibilité des mesures. Ce travail constitue un exemple de défi métrologique car les mesures énergétiques sur ces mélanges gazeux sont particulièrement délicates car complexes et également agressives pour l'instrumentation.

\section{Métrologie et Optoélectronique}

Un deuxième exemple de développement en cours sur la plateforme MATIS concerne la métrologie des propriétés thermiques des matériaux aux échelles micrométriques et sub-micrométriques. Plusieurs besoins motivent ces travaux scientifiques.

Tout d'abord se préparer à la demande émergente de traçabilité des mesures des propriétés thermo-physiques des matériaux aux très faibles échelles dimensionnelles, d'autre part mieux appréhender la métrologie et la physique à ces échelles pour répondre à des applications/besoins industriels nouveaux tels que le comportement thermique des matériaux sans cesse plus complexes utilisés en optoélectronique.

Ces développements ont conduit d'une part à la réalisation d'une thèse doctorale pour étudier les transferts et propriétés thermiques dans les nouvelles générations de couches minces employées comme support de stockage de l'information (mémoires) [6].

L'objectif de ces travaux était de contribuer à construire une installation de mesure de référence dédiée à la caractérisation thermique à l'échelle micrométrique d'un alliage particulier à base de tellure $\left(\mathrm{Ge}_{2} \mathrm{Sb}_{2} \mathrm{Te}_{5}\right)$ lorsque ce matériau se trouve à l'état fondu à haute température. C'est le changement d'état qui est exploité pour stocker par exemple une information numérique. La qualité et la durabilité des données enregistrées par ce procédé impose de maîtriser notamment la variation des propriétés de transport thermiques comme la conductivité thermique.

Les principes fondamentaux de la physique liés à la mise en œuvre de la technique de Radiométrie Photothermique modulée ont été étudiés et développés en collaboration avec l'Université de Bordeaux, Laboratoire Trèfle sous la Direction du professeur J.-L. Battaglia. Cette technique a permis d'estimer la résistance thermique de dépôt, de l'ensemble des interfaces à partir d'un modèle d'étude des transferts thermiques exploitant le formalisme des impédances thermiques. La conductivité thermique a pu être étudiée de la température ambiante jusqu'à $400^{\circ} \mathrm{C}$.

Ces travaux nous ont amenés également à entreprendre des collaborations sur des thématiques connexes avec les meilleurs instituts nationaux de métrologie mondiaux comme l'AIST-NMIJ au Japon.

Figure 3. Installation de référence du LNE pour l'étude des transferts thermiques dans les couches minces

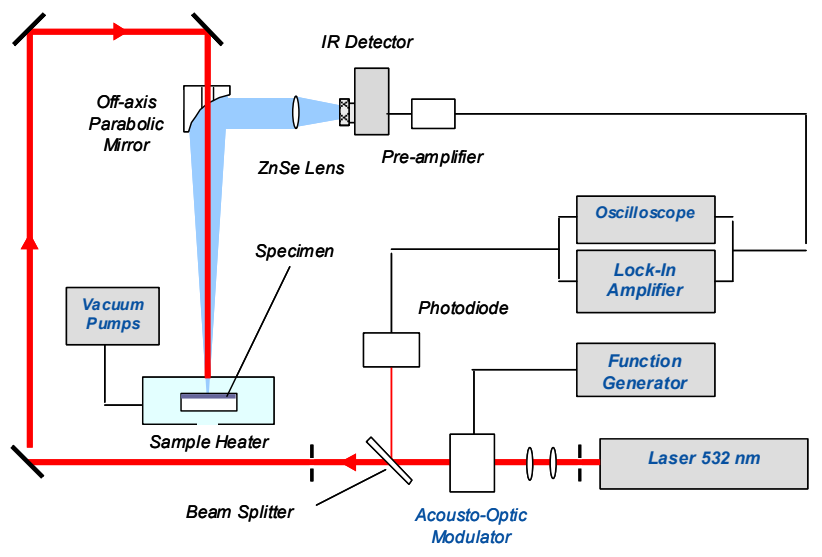

Par ailleurs la qualification métrologique de cette installation a été entreprise et se poursuit dans le cadre de la réalisation $\mathrm{du}$ projet IND07 «Thin Films» du programme européen de recherche en métrologie (EMRP), dont le LNE est partenaire. [7,8]

\section{En résumé}

Une plate-forme de caractérisation thermique et optique des matériaux utiles aux besoins exprimés par l'industrie et la société (MATIS) est maintenant opérationnelle sur le site du LNE à Trappes. Cet ensemble réunit les équipes d'experts du Laboratoire Commun de Métrologie du LNE et du CNAM.

Ces moyens en évolution technique permanente complètent les compétences déjà existantes ou en construction au LNE dans les domaines électrique, électromagnétique, chimique, mécanique et thermique. 
Ils visent à répondre aux enjeux suivants :

- Industriel : garantir la fiabilité des mesures des propriétés physico-chimiques des matériaux de la conception à l'utilisation de systèmes,

- Société : contribuer à l'établissement de fiches d'identité des matériaux afin de préciser leurs performances ou à prévenir les risques liés à leur utilisation,

- Scientifique : rechercher, étudier et développer de nouvelles techniques de mesures permettant de caractériser des matériaux de plus en plus complexes

Le LNE propose ainsi des capacités techniques et scientifiques toujours plus complètes et performantes au sein d'une infrastructure métrologique européenne de la mesure au conseil pour :

- concevoir des solutions industrielles dédiées et associées à du conseil ou de la formation,

- mesurer des propriétés des matériaux traçables au Système International d'unités en bénéficiant des atouts d'une implication forte dans une infrastructure métrologique européenne en construction,

- développer des méthodes et des instruments innovants et toujours plus précis pour mesurer les propriétés thermiques et optiques des matériaux, à l'échelle de quelques centaines de nanomètres jusqu'à plusieurs centimètres, sur une large gamme de températures et de longueur d'onde.

\section{Remerciements}

Le programme de recherche en métrologie européen "EMRP" est conjointement financé par les pays membres d'Euramet et l'Union Européenne.

Que le personnel du laboratoire impliqué dans les sujets qui ont été abordés dans cet article et notamment $\mathrm{F}$. Haloua, E. Foulon, A. Cappella, G. Davée et N. Fleurence sans oublier tous les autres collègues des équipes thermiques et optiques, soient vivement remerciés pour les nombreuses expérimentations complexes réalisées en équipe avec enthousiasme, efficacité et professionnalisme au sein de la plateforme MATIS

\section{Références}

1. "Development of a Reference Calorimeter and Proof of $0.05 \%$ Uncertainty”, Final report, GERG Project 1.44, (2010)
2. P. Schley, M. Beck, M. Uhrig, S. M. Sarge, J. Rauch, F. Haloua, J.-R. Filtz, B. Hay, M. Yakoubi, J. Escande, A. Benito, P. L. Cremonesi, "Measurements of the Calorific Value of Methane with the New GERG Reference Calorimeter" DOI 10.1007/s10765-010-0714z, Int J Thermophys, (2010)

3. F. Haloua, T. Macé, B. Hay, J.-R. Filtz, C. Graciannette, A. Royer, Ch. Sutour, F. Marioni, G. Hervouët, "Développement d'un calorimètre de référence pour la mesure du pouvoir calorifique des composants du gaz naturel avec une incertitude de +:$0,05 \% »$, Rapport final, (2004)

4. "ENG01 GAS: Characterisation of Energy Gases", EMRP 2009, Joint Research Project Protocol, Annex Ia, Euramet, (2010)

5. Rapport d'Activité 2012 du Laboratoire Commun de Métrologie du LNE-CNAM, Pôle PhotoniqueEnergétique, (2013)

6. A. Cappella, "Caractérisation thermique à haute température de couches minces pour mémoires à changement de phase depuis l'état solide jusqu'à l'état liquide », Ecole Doctorale des Sciences Physiques et de l'Ingénieur, Université de Bordeaux, n4500,(2012)

\section{7. http://projects.npl.co.uk/optoelectronic films/}

8. Publishable JRP Summary Report for JRP IND07 (ThinFilms) "Metrology for the manufacturing of thin films "http://www.euramet.org/fileadmin/docs/EMRP/JRP /JRP_Summaries_2010/IND07_Publishable_JRP_Summ ary.pdf, (2012) 